\title{
Rationality of utilization of antimicrobial agents in medical intensive care unit of a tertiary care hospital
}

\author{
Krishnendu Mondal*, Suvadip Biswas, Aritra Ghosh, Saikat Kumar Dalui, \\ Angana Datta, Supreeti Biswas
}

Department of Pharmacology, Burdwan Medical College, Burdwan, West Bengal, India

Received: 21 July 2016 Accepted: 23 August 2016

*Correspondence to: Dr. Krishnendu Mondal, Email: mondal.krishnendu367@ gmail.com

Copyright: (C) the author(s), publisher and licensee Medip Academy. This is an openaccess article distributed under the terms of the Creative Commons Attribution NonCommercial License, which permits unrestricted noncommercial use, distribution, and reproduction in any medium, provided the original work is properly cited.

\begin{abstract}
Background: Patients admitted to intensive care unit receive multiple medications of different pharmacological classes due to various life threatening ailments. This study was conducted to assess the patterns of usage of antimicrobial agents in medical ICU of a tertiary care hospital and to suggest necessary modifications in prescribing patterns to achieve rational therapeutic practices.

Methods: A cross-sectional observational study was carried out at ICU of the tertiary care hospital for 6 months. From the inpatient case record of ICU relevant data on prescription of each patient was collected. The demographic status, disease data and the utilization of different antimicrobial drug classes and individual drugs were analysed.

Results: Of 753 patients admitted in the medical ICU during the study period, 640 consecutive patients were included for analysis. Male to female ratio was 1.45. Mean age was $63.32 \pm 17.93$ years. Extensive poly-pharmacy $(100 \%)$ and drugs with non-generic name $(73 \%)$ noticed among the prescriptions.Average number of drugs per prescription was $12.1 \pm 2.13$. Penicillins $(51.87 \%)$ and cephalosporins $(45.78 \%)$ were most commonly used antimicrobial drug classes. Piperacillin (37.03\%), ceftriaxone $(33.28 \%)$ and levofloxacin $(22.5 \%)$ were commonly used antimicrobial drugs. A total of 181 prescriptions contained two and 138 contained three antimicrobial drugs. Piperacillin+tazobactam(37.03\%) was the most common fixed dose combination noticed.

Conclusions: Overall extensive poly-pharmacy and drugs with non-generic name noticed among the prescriptions. Few interventional programs should be aimed at control of infections, rational antimicrobial drug prescription to minimize adverse drug events, emergence of bacterial resistance and attenuating unnecessary cost.
\end{abstract}

Keywords: Rationality, Antimicrobial agents, Intensive Care Unit, Drug resistance

\section{INTRODUCTION}

Drug utilization research is defined as marketing, distribution, prescription and use of drugs in a society, with special emphasis on resulting medical, social and economic consequences. ${ }^{1}$ Drug utilization study is an essential part of pharmaco epidemiology. It is an important measure to study the clinical use of drugs in population and its impact on health-care system. ${ }^{1,2}$ Antimicrobial drug utilization study is very essential in an intensive care unit (ICU) setting because critically ill patients are often admitted in the ICUs who are usually exposed to multiple invasive procedures, and prone to multidrug resistant pathogens so why multiple broad spectrum antibiotics are prescribed empirically at admission on the basis of physician comfort and prior experience. But this overuse or misuse of antibiotics increases burden of antibiotic resistance, adverse effects of these drugs along with treatment costs and it's an important problem influencing patient outcomes. ${ }^{3-5,6}$ Multidrug-resistant clones is an emerging issue and as those can be treatable only by few limited available newer antibiotics emphasizing the urgent need for stringent infection control practices, vigilant surveillance as well as rational antibiotic prescription. ${ }^{3,47}$ Continuous injudicious and overt use of antimicrobial agents 
promoting emergence of antibiotic-resistant organisms have been reported by several authors. ${ }^{8,9}$ Rational use of drugs is defined as patients receiving medications appropriate to their clinical needs, in doses that meet their individual requirements, for an adequate period of time, and at lower cost to them and their community. ${ }^{10}$ Worldwide emergence of bacterial resistance, increased adverse effects and cost of the treatment can be effectively controlled by rational use of antimicrobial agents. $^{11,12}$ Fundamental knowledge about prescribing antibiotics is essential to obtain rational utilization. The information on the past performance of the antimicrobial agent prescribers and consumers is the mainstay in all the auditing systems. ${ }^{13}$

Regarding such usage of antimicrobials, we had planned to study the prescribing and utilization pattern and aimed to analyse rationality of usage of antimicrobial agents administered to the patients admitted in medical ICU of Burdwan medical college and Hospital, West Bengal.

\section{METHODS}

This study was an observational and cross-sectional study conducted at the ICU of Burdwan Medical College and Hospital (a tertiary care Govt. Medical College Hospital in West Bengal) over a period of 6 months $\left(23^{\text {rd }}\right.$ May 2015 to $22^{\text {nd }}$ November 2015 ).

This study was done in the patients admitted in the intensive care unit of the hospital during the study period of those six months. All the patients admitted in the ICU were included in the study. 640 patients were selected based on the criteria. The ICU was visited on every day during the study period and information about the patient demographics and drug used were recorded in a semistructured proforma.

\section{Inclusion criteria}

All patients admitted in the Medicine ICU

\section{Exclusion criteria}

- Patients stayed for less than 24 hours

- Patients with incomplete data

\section{Patient data collection form}

Data was obtained by using a self-designed data collection form, which includes details like patient demographics, laboratory data, drug treatment details and other relevant information

\section{Patient medical record}

Data was obtained from the inpatient case records of patients admitted in the ICU which comprised of patient demographic status, diagnosis given in the discharge summary, drugs used per prescription, duration of hospitalization, patient outcome following hospitalization e.g transferred to the general ward, discharged or referred to higher centres for further management etc.

\section{The parameters studied were}

- Most common causes for admission in ICU

- Average age of patients admitted

- Male and female patient ratio

- Most commonly used antimicrobials

- Average number drugs prescribed per patient

- Other drugs commonly used in ICU

- Drugs used in generic names

- Outcome of the patients.

Relevant data of the aforesaid study parameters were obtained, mean \pm SD number of drugs was calculated and finally data analysis was done using Graph Pad Instat 3.0 (trial version), Graph Pad Software Inc. 7825 Fay Avenue, Suite 230 LaJolla, CA, 92037 USA.

\section{RESULTS}

A total of 753 patients admitted in the medical ICU during the study period of 6months, 640 consecutive patients were included for analysis.

Out of 640 patients, highest number of 220 patients $(34.375 \%)$ was in the age group 71-80 years (Table 1$)$.

Table 1: Demographic profile of patients according to different age groups $(\mathrm{N}=640)$.

\begin{tabular}{|llll|}
\hline Age group & $\begin{array}{l}\text { Number } \\
(\%)\end{array}$ & $\begin{array}{l}\text { Sex ratio } \\
(\mathbf{M}: \mathbf{F})\end{array}$ & $\begin{array}{l}\text { Mean age } \\
\text { (years) }\end{array}$ \\
\hline 11-20 years & $21(3.28)$ & $13: 8$ & \\
\hline 21-30 years & $23(3.59)$ & $16: 7$ & \\
\hline 31-40 years & $37(5.78)$ & $25: 12$ & \\
\hline 41-50 years & $31(4.84)$ & $21: 10$ & \\
\hline 51-60 years & $135(21.09)$ & $78: 57$ & $63.32 \pm 17.93$ \\
\hline 61-70 years & $102(15.93)$ & $63: 39$ & \\
\hline 71-80 years & $220(34.37)$ & $121: 99$ & \\
\hline 81-90 years & $67(10.46)$ & $39: 28$ & \\
\hline$>90$ years & $4(0.625)$ & $3: 1$ & \\
\hline
\end{tabular}

Mean age was $63.32 \pm 17.93$ years. In all age groups, male preponderance was higher. Male to female ratio was 1.45. A wide spectrum of clinical diagnosis was observed including ischemic heart diseases (unstable angina and myocardial infarction), cerebro vascular accidents (CVA), acute exacerbation of COPD, acute left ventricular failure, different types of cardiac arrhythmias, acute kidney injury in case of chronic renal failure and cirrhosis of liver. Out of 640 patients, highest no. of patients (28.91\%) was admitted for ischemic heart diseases, followed by patients admitted for CVA (17.34\%). It was observed that male patients outnumbered female patients in all disease conditions (Table 2). 
189 patients were hospitalized for a time period less than 3 days, 230 for a time period between 3 to 6 days and 221 patients for a time period greater than 6 days.

Table 2: Demography for clinical diagnosis of the patients $(N=640)$.

\begin{tabular}{|c|c|c|c|c|c|}
\hline \multirow[t]{2}{*}{ Disease } & \multirow[t]{2}{*}{ Number (percentage) } & \multirow[t]{2}{*}{ Male to female ratio } & \multicolumn{3}{|c|}{$\begin{array}{l}\text { Number of patients with average } \\
\text { hospitalization period of }\end{array}$} \\
\hline & & & $<3$ days & 3-6 days & $>6$ days \\
\hline Ischemic heart disease & $185(28.91 \%)$ & 1.28 & 45 & 82 & 58 \\
\hline CVA & $111(17.34 \%)$ & 1.59 & 27 & 33 & 51 \\
\hline Acute ex. of COPD & $106(16.56 \%)$ & 1.65 & 25 & 32 & 49 \\
\hline Acute LVF & $91(14.21 \%)$ & 1.24 & 29 & 39 & 23 \\
\hline Cardiac arrhythmias & $57(8.90 \%)$ & 1.48 & 26 & 18 & 13 \\
\hline $\mathrm{AKI}$ in case of CRF & $49(7.65 \%)$ & 1.88 & 28 & 12 & 9 \\
\hline Cirrhosis of liver & $41(6.40 \%)$ & 1.56 & 9 & 14 & 18 \\
\hline
\end{tabular}

Drug therapies were categorized according to indication for the antimicrobial use. ${ }^{11}$ Physicians defined three usage groups according to the way they treated the patients.

- If clinical and/or laboratory data gave evidence, infection was considered as the indication.

- If there was no evidence of infection and the antibiotic was employed to prevent infection (e.g. catheterization), the therapy was considered as prophylactic.

- If no evidence of prophylaxis could be found, no direct evidence of infection present and records show the same symptoms being treated then indication of antimicrobial use was considered as symptomatic.

The common indications for antimicrobial use in our study were infection (61\%) followed by symptomatic (27\%) and prophylactic (12\%) (Table 3).

Table 3: Indication and rationality for antimicrobial use $(\mathrm{N}=640)$.

\begin{tabular}{|ll|}
\hline \multirow{2}{*}{ Indication of AMA use } & Infection (61\%) \\
\hline \multirow{3}{*}{ Rationality for AMA use } & Prophylactic (12\%) \\
\hline & Rational (23\%) \\
\cline { 2 - 2 } & Irrational (63\%) \\
\hline & Questionable (14\%) \\
\hline
\end{tabular}

\section{Rationality of antimicrobial use classified as ${ }^{11}$}

- The therapy was considered rational if the antimicrobial use and its route of administration, frequency, dose and duration of use were considered as accurate for infection.

- Therapy was considered irrational if the antimicrobial was used without specific indication, prophylaxis under circumstances of unproven efficacy or by clearly inappropriate route, dose or preparation for that indication.

- Therapy was considered questionable when sufficient clinical or laboratory data was unavailable to establish the therapy to be classified as clearly rational or irrational e.g patient with congestive heart failure having productive cough but do not know that cough is due to infection or the heart failure itself then treatment with antimicrobial agent considered questionable.

In this study $23 \%$ of AMAs were rational, $63 \%$ irrational and $14 \%$ questionable (Table 3 ).

Penicillins (51.87\%) and cephalosporins (45.78\%) were most commonly used antimicrobial drug classes, followed by fluoroquinolones (35.94\%), carbapenems (18.75\%), aminoglycosides (10.94\%) and others (6.37\%) (Figure 1).

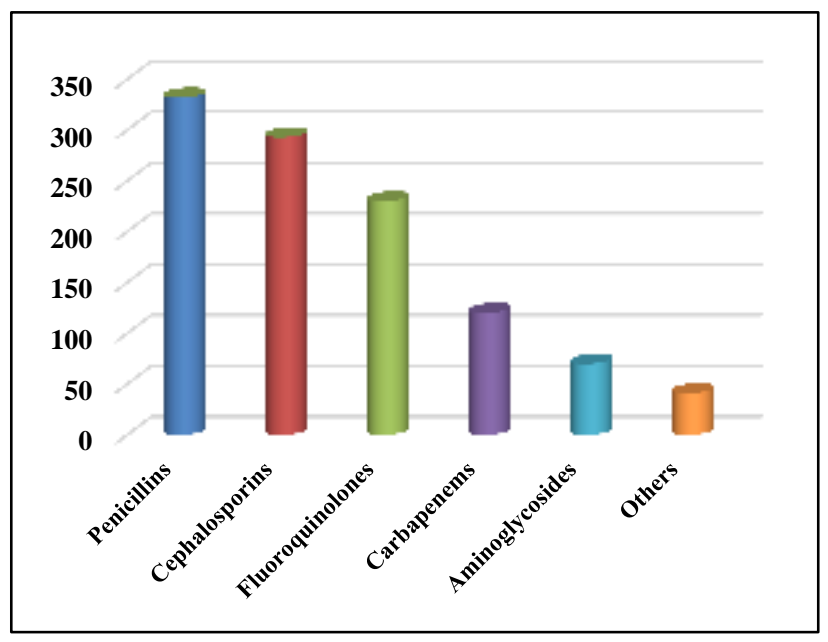

Figure 1: Most commonly used antimicrobial drug classes. 
Among all the individual antimicrobial drugs piperacillin $(37.03 \%)$, ceftriaxone $(33.28 \%)$ and levofloxacin $(22.5 \%)$ were most commonly used. Among penicillins, piperacillin was given in $71.28 \%$ cases and amoxycillin in $28.72 \%$ cases. Among cephalosporins, ceftriaxone was used in $72.69 \%$ cases and cefoperazone in $27.31 \%$ cases (Figure 2).

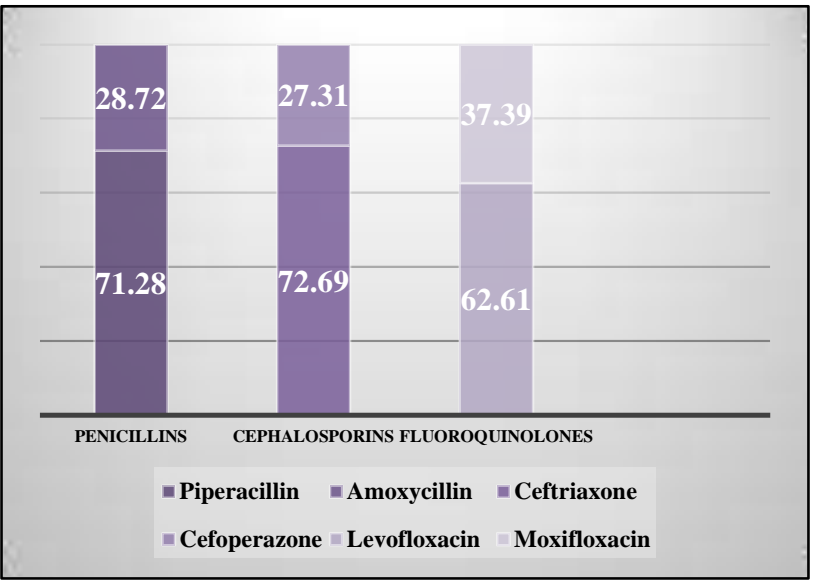

Figure 2: Most commonly used individual antimicrobial agents.

A total of 181 prescriptions contained two and 138 contained three antimicrobial drugs. Piperacillin+Tazobactam $(37.03 \%)$ were the most common fixed dose combination (FDC) noticed followed by Ceftriaxone+Sulbactum (31.09\%) (Figure 3 ).

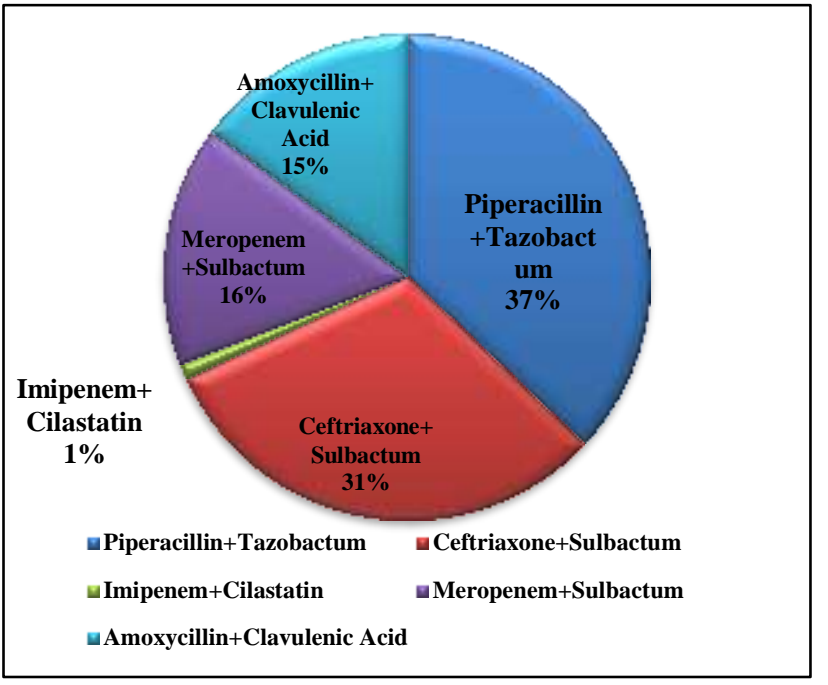

Figure 3: Most commonly used fixed dose combination antimicrobials.

Extensive poly-pharmacy (100\%) and drugs with nongeneric name $(73 \%)$ noticed among the prescriptions. Average number of drugs per prescription was $12.1 \pm 2.13$. It was observed that majority of patients $(59.53 \%)$ received $>7$ drugs followed by $(23.59 \%)$ patients received less than or equal to 7 drugs.
Most commonly used other drugs were pantoprazole (100\%), ondansetron (93.37\%), aspirin (67.32\%), clopidogrel, atorvastatin, glyceryl trinitrate, fondaparinux, LMWH, tramadol, midazolam, digoxin, ACE inhibitors, AT1 antagonists, beta blockers, furosemide, hydrocortisone, atropine, mannitol, dopamine, dobutamine, noradrenaline, amiodarone, phenytoin, levetiracetam, salbutamol, doxophylline, adenosine etc.

The maximum and minimum numbers of drugs prescribed to a single patient were 16 and 5 respectively. Improvement was seen in $85.36 \%$ patients while mortality was observed in $2.07 \%$ of patients and condition remained same in $12.57 \%$ patient at the time of discharge.

\section{DISCUSSION}

Ischemic heart disease was the common diagnosis among the patients admitted in the ICU in the present study.

Multiple antimicrobial agents and cardiovascular drugs were administered to the patients. $74 \%$ of patients admitted to a medical ICU were treated with antimicrobial medication according to the study done by Hanssens et al. ${ }^{14}$ A prospective antibiotic utilization survey done in two different medical departments showed $35.3 \%$ and $39 \%$ of the acute admitted patients having at least one antimicrobial exposure. ${ }^{15}$

The commonest AMA prescribed was piperacillin, this is in contrast to a similar study where most common antimicrobial agent used was ceftriaxone $(57 \%)$ as initial therapy,14whereas ampicillin, amoxicillin, metronidazole, ciprofloxacin, crystalline penicillin were the most commonly prescribed five antibiotics in the study conducted by Shankar et al. ${ }^{16}$

In ICU the condition of the patients are always critical so they receive parental route to overcome the emerging life threatening condition. Infection was the common indication for antimicrobial therapy in this study; supported by similar study where patients treated for presumed or proven infections were $76 \%$ and received antibiotics. $^{14} \mathrm{~A}$ high percentage of patients i.e. $87 \%$ patients was prescribed minimum one antibiotic which was similar with study done by Hanssens Yet al $(76 \%)$ and Shankar et al (92\%) in a teaching hospital of western Nepal but in contrast to van der Meer JW et al which shown $30 \%$ were prescribed antibiotics during the study period. $^{14,17,18}$ The variation in average percentage of patients receiving at least one antibiotic, which was $41 \%$, $45 \%, 79 \%$ and $98 \%$ in different health centers was shown by the study done by Bosu et al. ${ }^{19}$ As the patients are not matched socio- economically, so any firm conclusion can't be drawn. In this study patients received more than one AMA on various occasions. Patients receive one antimicrobial drug for gram positive, one for gram negative and another one for anaerobic infection because these patients were suffering from mixed infections. Patients received alternate antibiotics one by one many 
times when first one is not effective without doing culture sensitivity tests.

Prescription of a well-documented drug at an adequate dose, along with the correct information, at an affordable price determines rational use of a drug. Continuous, excessive and indiscriminate use of antimicrobial agents promoting the emergence of antibiotic-resistant organisms 20 had been expressed by a study done by Krivoy $\mathrm{N}$ et al. In this study $23 \%$ of AMAs were rational, $63 \%$ irrational and $14 \%$ questionable which is in accordance with a study conducted by Badar VA and Navale SB that showed that $30 \%, 60 \%$ and $11 \%$ of the AMAs were rational, irrational and questionable respectively. ${ }^{21}$

Effective reduction of antibiotic costs by $51 \%$ by a clinical pharmacologist was demonstrated by a prescription-point prevalence analysis when it was done for comparison between two internal medical departments. $^{15}$

The indications for antimicrobial utilization was infection $(61 \%)$ followed by symptomatic $(27 \%)$, prophylactic $(12 \%)$. The percentage of patients treated for infections was in accordance with $58.5 \%$ reported by other studies. ${ }^{22}$ The percent of prophylactic treatment prescribed is $12 \%$ which is similar to $13 \%$ and $10.3 \%$ reported in previous studies. ${ }^{18,19}$ In our study average number of drugs per prescription was $12.1 \pm 2.13$ which is in accordance to a similar study where the number was $12.1 \pm 7.6 .^{23}$ Important index of prescription audit is average number of drugs per person. To avoid the increased risk of development of bacterial resistance, drug interaction, increased hospital cost; it is required to restrict the number of drugs per prescription as low as possible. ${ }^{24}$ Physicians must be aware of the prevalence of different pathogens and resistance patterns in their hospital, have a clear understanding of therapeutic use of antibiotics and use empirical antibiotic regimens sensibly. ${ }^{14}$

\section{CONCLUSIONS}

Antibiotic resistance leads to increased morbidity, mortality and therapy cost. Inappropriate use of antibiotics is one of the main factors in the development of an antibiotic resistance. The physicians needs to understand that antibiotics are precious and of finite resources. Multidrug resistant organism not treatable by ever known antibiotic may emerge if no conscious efforts are made to contain the problem of drug resistance. So we all have to come together and find the remedy of this situation such as regulation of policies, education to both health care providers and to the patients and doing few interventional programs aimed at control of infections, rational antimicrobial drug prescription to minimize adverse drug events, emergence of bacterial resistance and attenuating unnecessary cost.

\section{ACKNOWLEDGEMENTS}

The authors wish to extend their sincerest thanks to Dr. Mithilesh Haldar, Dr. Subha Shankar Chattopadhyay for their enormous support to carry out the above research work. Sincere thanks are also to all the staffs in the ICU including attending doctors, nurses, laboratory technicians for their participation in the study.

\section{Funding: No funding sources \\ Conflict of interest: None declared \\ Ethical approval: Not required}

\section{REFERENCES}

1. WHO. The selection of essential drugs. WHO Technical report. 1977;615:36.

2. Sachdeva PD, Patel BG. Drug utilization studies scope and future perspectives. Int J Pharm Biol Res. 2010;1:11-7.

3. Esposito S, Leone S. Antimicrobial treatment for intensive care unit (ICU) infections including the role of the infectious diseases specialist. Int J Antimicrob Agents. 2007;29:494-500.

4. Lockhart SR, Abramson MA, Beekman SE, Gallagher G, Riedel SR, Diekma DJ, et al. Antimicrobial resistance among gram-negative bacilli as causes of infections in intensive care unit patients in the United States between 1993 and 2004. J Clin Microbiol. 2007;45:3352-9.

5. Weber RJ, Kane SL, Oriolo VA, Saul M, Skledar SJ, Dasta JF. Impact of intensive care drug costs: a descriptive analysis, with recommendations for optimizing ICU pharmacotherapy. Crit Care Med. 2003;31:17-24.

6. Curcio DJ. On behaf of the Latin American antibiotic use in intensive care unit group. Antibiotic prescription in intensive care units in Latin America. Rev Argent Microbiol. 2011;43:203-11.

7. Paterson DL, Rogers BA. How Soon Is Now? The urgent need for randomized, controlled trials evaluating treatment of multidrug-resistant bacterial infection. Clin Infect Dis. 2010;51:1245-7.

8. Niederman MS. Appropriate use of antimicrobial agents: challenges and strategies for improvement. Crit Care Med. 2003;31:608-16.

9. Pulcine C, Pradier C, Samat-Long C, Hyvernat H, Bernardin $\mathrm{G}$, Ichai $\mathrm{C}$, et al. Factors associated with adherence to infectious diseases advice in two intensive care units. J Antimicrob Chemother. 2006;57:546-50.

10. The rational use of drugs, report on the conference of experts Nairobi. Sponsored by WHO Geneva; 1985:25-29.

11. Goossens H. Antibiotic consumption and link to resistance. ClinMicrobiol Infect. 2009;15(Suppl 3):12-5.

12. MacDougall C, Polk RE. Antimicrobial stewardship programs in health care systems. Clin Microbiol Rev. 2005;18(4):638-56. 
13. Harbarth S, Harris AD, Carmeli Y, Samore MH. Parallel analysis of individual and aggregated data on antibiotic exposure and resistance in gram-negative bacilli. Clin Infect Dis. 2001;33(9):1462-8.

14. Hanssens Y, Ismaeili BB. Antibiotic prescription pattern in a medicalintensive care unit in Qatar. Saudi Med J. 2005;26:1269-76.

15. Gendel I, Azzam ZS, Braun E, Levy Y, Krivoy N. Antibioticutilization prevalence: prospective comparison between two medical departments in a tertiary care university hospital. Pharmacoepidemiol Drug Safety. 2004;13:735-9.

16. Shankar RP, Partha P, Shenoy NK, Easow JM, Brahmadathan KN. Prescribing patterns of antibiotics and sensitivity patterns of common microorganisms in the Internal Medicine ward of a teaching hospital in Western Nepal: a prospective study. Ann Clin Microbiol Antimicrob. 2003;2:7.

17. Shankar PR, Partha P, Dubey AK, Mishra P, Deshpande VY. Intensive care unit drug utilization in a teaching hospital in Nepal. Kathmandu University Medical Journal. 2005;3:130-7.

18. Van der Meer JW, Gyssens IC. Quality of antimicrobial drug prescription in hospital. Clin Microbiol Infect. 2001;7Suppl6:12-5.
19. Bosu WK, Ofori-Adjei D. Survey of antibiotic prescribing pattern in government health facilities of the was a west district of Ghana. East African Medical Journal. 1997;74:139.

20. Krivoy N, El-Ahal WA, Bar-Lavie Y, Haddad S. Antibiotic prescription and cost patterns in a general intensive care unit. Pharmacy Practice. 2007;5:67-73.

21. Badar Vandana A, Navale Sanjakumar B. Study of prescribing pattern of antimicrobial agents in medicine intensive care unit of a teaching hospital in Central India. JAPI. 2012;60:20-3.

22. Suping HU, Xiuheng LIU, Peng Y. Assessment of antibiotic prescription in hospitalised patients at a Chinese University hospital; 2002.

23. Smythe MA, Melendy S, Jahns B, Dmuchowski C. An explor atory analysis of medication utilization in a medical intensive care unit. Crit Care Med. 1993;21:1319-23.

24. Stratton $\mathrm{CW} 4^{\text {th }}$, Ratner H, Johnston PE, Schaffner W. Focused microbiological surveillance by specific hospital unit: practical application and clinical utility. Clin Ther. 1993;15SupplA:12-20.

Cite this article as: Mondal K, Biswas S, Ghosh A, Dalui SK, Datta A, Biswas S. Rationality of utilization of antimicrobial agents in medical intensive care unit of a tertiary care hospital. Int $\mathbf{J}$ Basic Clin Pharmacol 2016;5:2168-73. 